BNL-112115-2016-JA

\title{
Static and Dynamic Optical Properties of La1-xSrxFeO3- $\delta$ : The Effects of A-site and Oxygen Stoichiometry
}

\author{
Sergey Y. Smolin \\ Sergey Y. Smolin†, Mark D. Scafettał, Amber K. Choquetteł, Matthew Y. Sfeir§, \\ Jason B. Baxter†, and Steven J. Maył \\ tDepartment of Chemical and Biological Engineering, Drexel University, Philadelphia, PA \\ $\ddagger$ Department of Materials Science and Engineering, Drexel University, Philadelphia, PA \\ $\S$ Center for Functional Nanomaterials, Brookhaven National Laboratory, Upton, NY
}

Submitted to the Journal of Chemistry of Materials

\author{
Center for Functional \\ Brookhaven National Laboratory
}

\author{
U.S. Department of Energy \\ Office of Basic Energy Sciences
}

Notice: This manuscript has been authored by employees of Brookhaven Science Associates, LLC under Contract No. DESC0012704 with the U.S. Department of Energy. The publisher by accepting the manuscript for publication acknowledges that the United States Government retains a non-exclusive, paid-up, irrevocable, world-wide license to publish or reproduce the published form of this manuscript, or allow others to do so, for United States Government purposes. 


\section{DISCLAIMER}

This report was prepared as an account of work sponsored by an agency of the United States Government. Neither the United States Government nor any agency thereof, nor any of their employees, nor any of their contractors, subcontractors, or their employees, makes any warranty, express or implied, or assumes any legal liability or responsibility for the accuracy, completeness, or any third party's use or the results of such use of any information, apparatus, product, or process disclosed, or represents that its use would not infringe privately owned rights. Reference herein to any specific commercial product, process, or service by trade name, trademark, manufacturer, or otherwise, does not necessarily constitute or imply its endorsement, recommendation, or favoring by the United States Government or any agency thereof or its contractors or subcontractors. The views and opinions of authors expressed herein do not necessarily state or reflect those of the United States Government or any agency thereof. 


\title{
Static and Dynamic Optical Properties of $\mathrm{La}_{1-x} \mathrm{Sr}_{x} \mathrm{FeO}_{3-\delta}$ : The Effects of A-site and Oxygen Stoichiometry
}

\author{
Sergey Y. Smolin ${ }^{\dagger} \neq$, Mark D. Scafetta ${ }^{\ddagger} \neq$, Amber K. Choquette ${ }^{\ddagger}$, Matthew Y. Sfeir ${ }^{\S}$, Jason B. Bax- \\ ter $^{\dagger}$, and Steven J. May* $\neq$ \\ ${ }^{\dagger}$ Department of Chemical and Biological Engineering, Drexel University, Philadelphia, PA 19104 \\ ${ }^{\ddagger}$ Department of Materials Science and Engineering, Drexel University, Philadelphia, PA 19104 \\ ${ }^{\S}$ Center for Functional Nanomaterials, Brookhaven National Laboratory, Upton, NY 11973 \\ ${ }^{*}$ Authors contributed equally.
}

Supporting information

ABSTRACT: Perovskite oxides are a promising material class for photovoltaic and photocatalytic applications due to their
visible band gaps, nanosecond recombination lifetimes, and great chemical diversity. However, there is limited under-
standing of the link between composition and static and dynamic optical properties despite the critical role these proper-
ties play in the design of light-harvesting devices. To clarify these relationships we systemically studied the optoelectronic
properties in $\mathrm{La}_{1-\mathrm{S}} \mathrm{Sr}_{x} \mathrm{FeO}_{3-\delta}$ epitaxial films, uncovering the effects of $A$-site cation substitution and oxygen stoichiometry.
Variable angle spectroscopic ellipsometry was used to measure static optical properties, revealing a linear increase in ab-
sorption coefficient at $1.25 \mathrm{eV}$ and a red-shifting of the optical absorption edge with increasing Sr fraction. The absorption
spectra can be similarly tuned through the introduction of oxygen vacancies, indicating the critical role that nominal Fe
valence plays in optical absorption. Dynamic optoelectronic properties were studied with ultrafast transient reflectance
spectroscopy, revealing similar nanosecond photoexcited carrier lifetimes for oxygen deficient and stoichiometric films
with the same nominal Fe valence. These results demonstrate that while the static optical absorption is strongly depend-
ent on nominal Fe valence tuned through cation or anion stoichiometry, oxygen vacancies do not appear to play a signifi-
cantly detrimental role in the recombination kinetics.

\section{INTRODUCTION}

Perovskite oxides, with the general chemical formula $A B \mathrm{O}_{3-\delta}$, have long been the center of intense materials research efforts due to their varied physical, electronic and magnetic properties. ${ }^{1-3}$ In recent years, increased attention has turned to the optical behavior of complex oxides with growing interest in their use for lightharvesting applications such as photocatalysis or photovoltaics..$^{4-12}$ Of particular importance for such applications, many perovskite oxides have band gaps in the visible range ${ }^{13,14}$ with nanosecond recombination lifetimes, ${ }^{15-17}$ are largely composed of non-toxic and earth abundant elements, and are chemically stable. Additionally, their physical properties are extremely tunable through chemical substitution, epitaxial strain, and heterostructuring. ${ }^{18,19}$

The valence and conduction bands of non- $d^{\circ}$ perovskite oxides are mainly derived from the corner-connected $\mathrm{BO}_{6}$ octahedra, with the nominal $B$-site valence playing a critical role in physical properties that depend on electronic structure. As most $A$-site cations are stable in only a single oxidation state, the $B$-site valence can be tuned through either heterovalent $A$-site substitution, for example $\mathrm{Sr}^{2+}$ for $\mathrm{La}^{3+}$, or by introducing oxygen vacancies, $\delta \neq 0$. Therefore, both $A$-site substitution and oxygen stoichiometry provide means to tune optoelectronic properties, including absorption coefficients and recombination lifetimes, which are essential in the design of photovoltaic and photocatalytic devices. ${ }^{20}$ While recent studies have reported ultrafast optical spectroscopy in select perovskite oxides, ${ }^{15-17,22,23}$ systemic investigations of how recombination lifetimes change with composition, structure, or $B$-site valence are lacking.

In this work, we investigate the effects of $A$-site substitution and oxygen content on the static and dynamic optical properties of epitaxial $\mathrm{La}_{1-x} \mathrm{Sr}_{x} \mathrm{FeO}_{3-\delta}$ thin films. The $\mathrm{LaFeO}_{3}$ end member is a semiconductor with a band gap of $\sim 2.4 \mathrm{eV}$, a recombination lifetime of $2 \mathrm{~ns}$, and a $d^{5} \mathrm{Fe}$ electronic configuration. ${ }^{13,17,24}$ Structurally, $\mathrm{LaFeO}_{3}$ is orthorhombic with an $a^{-} a^{-} c^{+}$pattern of $\mathrm{FeO}_{6}$ rotations at room temperature. Increased $\mathrm{Sr}$ alloying alters the crystal structure to rhombohedral ( $\left.a^{-} a^{-} a^{-}\right)$near $x=0.3$ and finally cubic $\left(a^{0} a^{0} a^{0}\right)$ near $x=0.8 .^{25,26}$ Additionally the substitu- 
tion of $\mathrm{Sr}$ for $\mathrm{La}$ adds holes to the hybridized $\mathrm{Fe}-\mathrm{O}$ bands, ${ }^{27,28}$ which can be simplistically described as an increase of the nominal Fe valence from $3+$ to $4+$. In oxygen stoichiometric materials $(\delta=0)$, the resistivity decreases exponentially with increasing $x$ from $x=0$ to $0.5 .^{29,30} \mathrm{Be}-$ tween $x=0.5$ and 0.9 , the system undergoes a nominal charge ordering transition near $200 \mathrm{~K}^{31-34}$ Eventually, at higher $\mathrm{Sr}$ fraction $(x>0.9)$, the material exhibits metallic conduction at all temperatures without transitioning into an insulating charge ordered phase. ${ }^{35}$ The addition of $\mathrm{Sr}$ has also been shown to induce new states within the gap of $\mathrm{LaFeO}_{3},{ }^{36}$ leading to increased optical absorption between $1-2 \mathrm{eV}$ with increased $\mathrm{Sr}$ fraction as previously observed for compounds with $x \leq 0.4 .^{37}$ However, the formation energy of oxygen vacancies in $\mathrm{LaFeO}_{3}$ decreases significantly upon alloying with $\mathrm{Sr},{ }^{38}$ creating a strong propensity for the LSFO system to be oxygen deficient, ${ }^{39-41}$ thereby driving Fe into the energetically favorable $3+$ state over the nominal $4+$ state. Electronically, the consequence of increasing $\delta$ in $\mathrm{SrFeO}_{3-\delta}$ and LSFO is increasingly insulating behavior, ${ }^{29,42,43}$ consistent with a reduction of the nominal Fe valence toward $3+$. However, oxygen vacancy induced changes to the optical properties of LSFO have yet to be reported in detail.

Here, we provide a systematic comparison of how the optical absorption and transient reflectivity spectra evolve with changes in nominal Fe oxidation state through two different mechanisms, the substitution of $\mathrm{Sr}^{2+}$ for $\mathrm{La}^{3+}$ and through the controlled introduction of oxygen vacancies. We find the spectral evolution with changing $\delta$ closely matches that obtained from films with varying $\mathrm{Sr}$ concentration. By comparing the static optical absorption spectra obtained from oxygen stoichiometric films, the relative oxygen content in oxygen deficient films is estimated. The effect of heterovalent $A$-site substitution and oxygen vacancies on carrier lifetimes is then studied by comparing the recombination dynamics from films with the same nominal Fe valence but with differing $x$ and $\delta$. We find similar kinetic behavior for a given Fe valence, obtained through either $A$-site substitution or the formation of oxygen vacancies, suggesting that recombination lifetimes are relatively robust against oxygen vacancies in ferrite perovskites.

\section{EXPERIMENTAL}

\subsection{Synthesis}

Thin film samples were deposited using an Omicron LAB-10 molecular beam epitaxy (MBE) system modified for oxides. The base pressure of the system with the sources at deposition temperature is below $1 \times 10^{-8}$ Torr. Epitaxial films were deposited simultaneously onto single side polished single crystal $\mathrm{SrTiO}_{3}$ (STO) and (LaA$\left.1 \mathrm{O}_{3}\right)_{0.3}\left(\mathrm{Sr}_{2} \mathrm{TaAlO}_{6}\right)_{0.7}$ (LSAT) substrates. The specific LSFO films containing $x=0.17$ and $x=0.36$ are the same films discussed in a previous publication which reports further details of the over-all deposition process. ${ }^{37}$ The earlier paper $^{37}$ reports slightly different compositions of $\mathrm{x}=0.15$ and $x=0.40$, initially estimated (within experimental error) based on X-ray diffraction (XRD) and calibration depositions. The compositions of these films have since been measured by Rutherford backscattering spectrometry (RBS) and the updated compositions are reported here. The LFO used in this study is the same from a more recent publication and the deposition details for that sample can be found in Ref. 24.

For all other films shown here, a deposition pressure of $\sim 1 \times 10^{-6}$ Torr in $\mathrm{O}_{2}$ was used. The growth temperature, between $550^{\circ} \mathrm{C}$ and $750^{\circ} \mathrm{C}$, was monitored optically in real time by a LumaSense Technologies IGA 50-LO plus pyrometer at the STO surface center. The co-deposition times ranged from 20-45 seconds per unit cell including 5 second intervals with all shutters closed between layers. The thickness of the films is approximately $40 \mathrm{~nm}$. Ex-situ post-growth anneals were carried out in a horizontal tube furnace using a two-step process: 4 hours at $650^{\circ} \mathrm{C}$ under flowing $\mathrm{O}_{2}$ followed by cooling to $200^{\circ} \mathrm{C}$ and introducing ozone at $5 \%$ concentration for 1 hour then cooling to room temp under the flowing oxygen/ozone mixture. ${ }^{30,44}$ This post-growth anneal is necessary to fully oxidize the Sr-containing films.

Oxygen loss was carried out by heating the oxygen stoichiometric film on a hotplate in air at $300^{\circ} \mathrm{C}$. A standard 4.5" conflat copper gasket was placed between the hotplate surface and the bottom, unpolished side of the sample, to aid in heat transfer. By placing the sample onto the heated copper ring on the hotplate surface for various lengths of time the oxygen content was modulated in steps with longer exposure time leading to more oxygen loss. ${ }^{43}$ Ellipsometry and visible transient reflectance spectroscopy were performed at each interval to characterize the static and dynamic optical properties as a function of oxygen content and Fe valence. For transient reflectance measurements with a near- infrared probe, the same heating experiment was performed but corresponding ellipsometry data was not taken between each heating interval.

\subsection{Characterization}

Composition and structure. Cation composition was measured using RBS at Rutgers University for films deposited on STO. Based on the c-axis, electrical measurements, and the optical characterization, the composition of films on LSAT and STO grown in the same run is expected to be within the experimental error for RBS. Additionally, the $A: B$ cation ratios are $1: 1$ within the error of the RBS measurement. We note that typical errors for the RBS determination of the $\mathrm{Sr}$ and Fe compositions are 4 and $3 \%$, respectively, in the films. In calculation of nominal Fe valence, we use the $\mathrm{Sr}$ fraction, assume $\delta \approx 0$ in the oxygen stoichiometric films and that any cation-site deficiency (deviation from $A: B=1$ ) is charge balanced by oxygen vacancies. X-ray diffraction and reflectivity were measured using a Rigaku SmartLab diffractometer. The reflectivity data and select diffraction patterns were mod- 
eled using the GenX program ${ }^{45}$ to accurately determine film thickness, roughness, and c-axis. When the film and substrate diffraction peaks were not overlapping, Gaussian fitting of the film's diffraction peak was sufficient for accurate $c$-axis determination and was in close agreement with GenX simulations as seen in the supplemental materials of Ref. 37. Previously, reciprocal space maps were used to confirm that LFO, of similar thickness to the films in this study, grown on STO is coherently strained. ${ }^{37}$ The linear $c$-axis versus cation composition and equivalent measured coherence lengths and film thickness suggest the films in this study are coherently strained.

Static optical properties. Static optical properties were characterized over the energy range of $1.25-5 \mathrm{eV}$ using a J.A. Woolam M-20ooU variable angle spectroscopic ellipsometer (VASE). VASE was carried out at four or five angles from $60-75^{\circ}$ to create a well-defined sample set for the fitting software. VASE data was fit and analyzed using the WVASE32 V.3.826 software package to extract the index of refraction $(n)$ and the extinction coefficient $(k)$ of the film from the film-substrate stack. The optical constants of the films were modeled in a point by point fashion, with the thickness fixed at the value obtained from $\mathrm{x}$ ray reflectivity to obtain the most accurate description of the optical absorption response of the material. The film/substrate and surface roughness determined from $x$ ray reflectivity is less than $1 \mathrm{~nm}$; and therefore, films were modeled with smooth interfaces to simplify simulations. The difference due to $1 \mathrm{~nm}$ roughness in the resulting spectra was minimal. Optical absorption data was determined using the relationship $\alpha=4 \pi k / \lambda$, where $\alpha$ is the absorption coefficient and $\lambda$ is the incident photon wavelength. The substrate optical properties were measured with VASE for every individual substrate immediately prior to deposition after heating at $200^{\circ} \mathrm{C}$ while curing the silver paint used for mounting substrates to the growth stub. This process was found to yield substrates with similar optical properties as exposing the substrates to growth conditions for $\sim 1$ hour.

Dynamic optoelectronic properties. The recombination dynamics of photoexcited electrons and holes were measured with ultrafast transient reflectance spectroscopy (Ultrafast Systems Helios Spectrometer). Samples were pumped at $4.0 \mathrm{eV}$ with $\sim 50 \mathrm{fs}$ pulse duration, and differential reflectance was probed over the first 3 nanoseconds after photoexcitation. Care was taken to measure recombination dynamics independently of optical absorption by exciting in a region where the absorption does not change dramatically with either $x$ or $\delta(4.0 \mathrm{eV})$ because this would maintain similar densities of photoexcited carriers between samples. Temporal resolution was 50 fs and delay time was controlled by an optical delay line. Spectral response was monitored from $0.9-3.8 \mathrm{eV}$ using a white light continuum probe.

For the UV-vis spectrometer, pulses were generated at 1 $\mathrm{kHz}$ repetition rate using a Coherent Libra HE Ti:sapphire regeneratively amplified laser and an optical parametric amplifier (OPA, Coherent OPerA Solo). The white light (1.8-3.8 eV) probe pulse was generated by focusing the $800 \mathrm{~nm}$ seed pulse onto a $\mathrm{CaF}_{2}$ crystal. Detection was performed with a Si CCD array. ${ }^{17}$ For the near-infrared spectrometer, the optical parametric amplifier (Light Conversion TOPAS-C) was pumped with a Spectra-Physics Spitfire Ti:Sapphire laser which outputs 110 fs pulses centered at $800 \mathrm{~nm}$ with a $1 \mathrm{kHz}$ repetition rate. The near-infrared probe (o.9-1.5 eV) was generated by focusing the $800 \mathrm{~nm}$ seed pulse into a sapphire crystal. Detection was performed by InGaAs multichannel detector.

\section{RESULTS AND DISCUSSION}

$$
\text { 3.1 Crystal quality }
$$
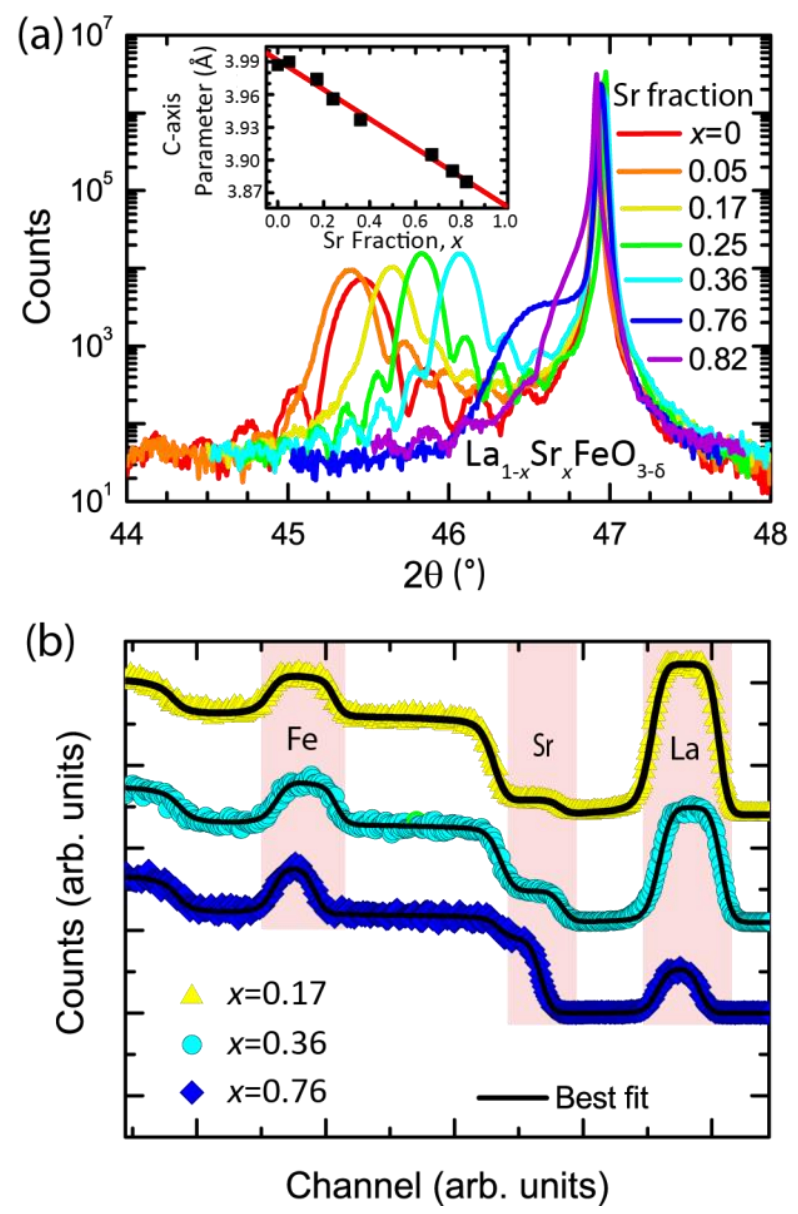

Figure 1. (a) X-ray diffraction of $\mathrm{La}_{1-\mathrm{x}} \mathrm{Sr}_{\mathrm{x}} \mathrm{FeO}_{3-\delta}$ films $(\delta \approx 0)$ on LSAT and corresponding c-axis crystal parameter versus $\mathrm{Sr}$ fraction (inset). (b) Rutherford backscattering spectrometry data and fits for three representative films; the data is offset for clarity. Red shading is an approximate representation of the region used for estimating cation composition within the films.

The film quality and cation stoichiometry were investigated using XRD and RBS. XRD and RBS data is presented in Figure 1(a) and (b), respectively, for a series of compositionally varied samples. RBS data and fits for the $x=0.25$ and 0.82 films can be found in Ref. 30. The XRD data is 

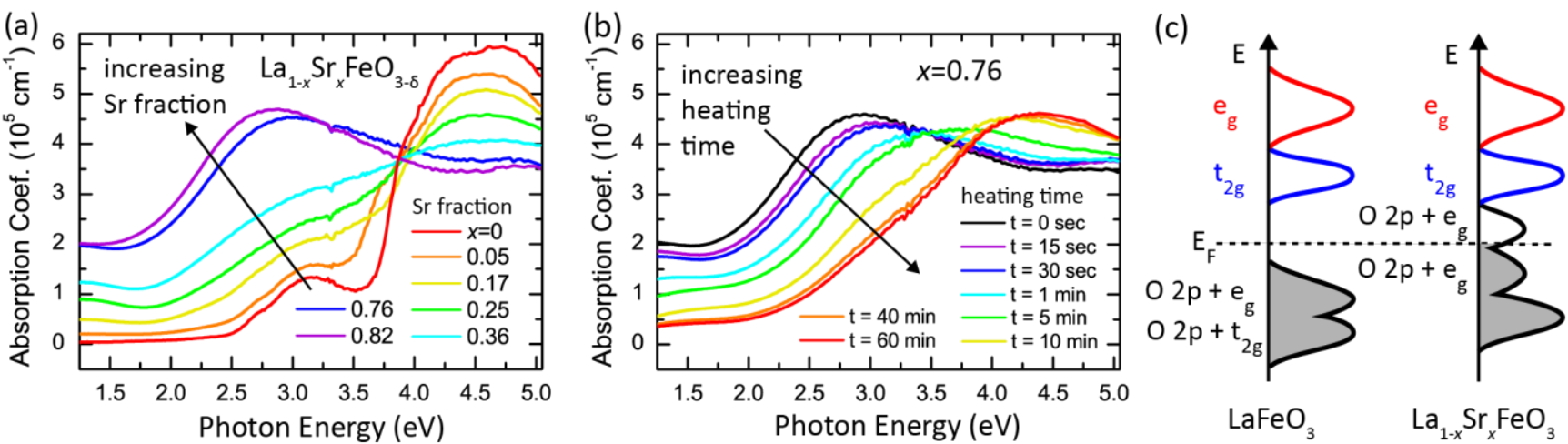

Figure 2. Optical absorption changes in LSFO with changing (a) Sr fraction $(\delta \approx 0)$ and (b) oxygen content. (c) Schematic density of states diagrams for LFO and LSFO, showing effect of Sr substitution. We hypothesize that similar changes are brought about through oxygen vacancies. The schematic in (c) is based on Refs. 24 and 36 with majority spin states outlined in black and minority spin states outlined in red and blue. The presence or absence of the band gap in (c) for LSFO is dependent on the Sr fraction.

from samples on LSAT measured after the post-growth annealing process. The measured pseudocubic c-axis parameters are shown inset of Figure 1(a); the in-plane lattice parameters are assumed to be equal to that of the LSAT substrate $(a=3.87 \AA)$. The error in the $c$-axis parameter is estimated to be no more than $\pm 0.005 \AA$. The origin of the slight lattice expansion in $x=0.05$ compared to $x=0$ is unknown. The broader and smaller intensity for the $x=0.76$ film peak is a result of the oxygen deficient film thickness $(=35 \mathrm{~nm})$ for that particular sample. This same film was used in subsequent oxygen loss experiments for its relative ease of oxidation/reduction and relatively high $\mathrm{Sr}$ concentration.

RBS data was taken from as-grown samples on STO. These samples were deposited in the same run as the corresponding films on LSAT. The composition for films grown in the same run is consistent within the experimental error of RBS, based on additional XRD, X-ray photoelectron spectroscopy (XPS), optical, and electrical measurements.

\subsection{Composition-dependent absorption}

Effect of $\boldsymbol{A}$-site composition. The optical absorption spectra measured from the oxygen stoichiometric LSFO films with varying $\mathrm{Sr}$ fraction on LSAT are shown in Figure 2 (a). Numerous systematic trends brought on by $\mathrm{Sr}$ substitution are observed in the spectra. Sr substitution induces a low energy $(<1.5 \mathrm{eV})$ absorption feature due to the formation of a new state below the bottom of the conduction band in the LFO parent material, consisting of $\mathrm{O} 2 p$ and majority spin Fe $e_{\mathrm{g}}$ orbitals. ${ }^{36}$ This new band associated with $\mathrm{Sr}$ substitution is created at the expense of valence band $\mathrm{O} 2 p$ and majority spin Fe $e_{\mathrm{g}}$ orbitals. ${ }^{36}$

There is an apparent redshift of the optical transition associated with the LFO band gap with increasing Sr substitution; in LFO this absorption edge is due to the excitation of electrons from near the valence band maximum into the conduction band, comprised mostly of unoccu- pied minority spin Fe $t_{2 \mathrm{~g}}$ states. ${ }^{24,36}$ This implies that the separation of the valence band and the band derived from minority spin $t_{2 g}$ states is decreasing with increasing $\mathrm{Sr}$ substitution, in agreement with valence band positions measured by Wadati et al. ${ }^{36} \mathrm{~A}$ previous study of LFO found that the band gap energy was most accurately determined by treating the transition using a direct forbidden Tauc model. ${ }^{24}$ The addition of Sr alters the absorption spectrum by enabling transitions into empty majority spin Fe $e_{g}$ states. The most appropriate Tauc model within which to analyze the absorption arising from these transitions is unknown. We note though that the significant increase in absorption coefficient below $3 \mathrm{eV}$ indicates that the transition becomes less "forbidden" with increasing $\mathrm{Sr}$ doping. However, for the sake of comparison to LFO the transition energy was determined from a direct forbidden Tauc model for the films and found to decrease linearly from $x=0$ to $x \sim 0.5$, as shown in the supporting information, Figure S1. Increased Sr substitution beyond $x=0.5$ results in an increasing slope of this absorption edge as seen in Figure 2(a), and the resulting transition energy (approximately $1.2 \mathrm{eV}$ ) is nearly independent of $x$ for $x>0.5$. Finally, the absorption associated with transitions between the valence band and unoccupied minority spin $e_{g}$ Fe states, above $\sim 3.5 \mathrm{eV}$, decreases with increasing $\mathrm{Sr}$ fraction. We speculate that this is due to the decreasing DOS at the valence band maximum with increasing $\mathrm{Sr}$ concentration.

Effect of oxygen vacancies. Given that nominal $B$-site valence is critically important to optical properties that depend on electronic structure, introducing oxygen deficiency into LSFO may have similar optoelectronic effects to heterovalent $A$-site substitution as both can alter the nominal Fe valence. Experimentally, changing the oxygen content is advantageous because oxygen fraction can be reversibly tuned within a single film without otherwise significantly altering crystal quality, cation stoichiometry, or thicknesses. Oxygen vacancies are easily induced in LSFO by exposure to elevated temperatures because it is 
energetically favorable for Fe to adopt the $3+$ valence state over the nominal $4+$ valence state..$^{38,43,46,47}$

We demonstrate the effect of oxygen vacancies on optical properties by measuring absorption of the same film after heating in air at $300^{\circ} \mathrm{C}$ for different times. Figure (b) shows the changes in optical absorption in the $x=0.76$ film with increasing cumulative heating time (oxygen loss). The changes in absorption spectra achieved by increasing heating time are very similar to those achieved by $A$-site substitution of La for Sr. Both substitution of La for $\mathrm{Sr}$ and formation of oxygen vacancies reduce the nominal Fe valence in LSFO, decreasing the number of unoccupied states above the Fermi level associated with the majority spin Fe $e_{g} / \mathrm{O}$ 2p orbitals.

Changes in the optical absorption spectra upon changing nominal Fe valence can be understood through the schematic of the density of states shown in Figure 2(c). This schematic is based on photoemission and x-ray absorption spectroscopy of LSFO reported in Ref. 36 and density functional theory calculations of LFO in Ref. 24. Substitution of $\mathrm{Sr}$ for La results in the redistribution of hybridized $\mathrm{O} 2 p / \mathrm{Fe} e_{g}$ states from the valence band to above the Fermi level. ${ }^{36}$ With sufficiently high $\mathrm{Sr}$ fraction, the room temperature band gap between the valence band and the new $\mathrm{Fe}^{4+}$-induced states closes; ${ }^{48}$ however, electronic transport remains semiconductor-like due to polaronic effects. ${ }^{34}$ The exact $\mathrm{Sr}$ fraction to which a band gap persists is unknown, although it is reasonable to speculate that $x=0.5$ marks the critical composition given the changes in electronic behavior observed near halfdoping. ${ }^{30}$ We believe that similar changes to the density of states arise when nominal Fe valence is manipulated by introducing oxygen vacancies.

Quantitative correlation between absorption and composition. The magnitude of the absorption coefficient at $1.25 \mathrm{eV}$ scales linearly with increasing $\mathrm{Sr}$ fraction for oxidizing films, as shown in Figure 3(a). This linear relationship serves as a convenient estimator of the $\mathrm{Fe}$ oxidation state in LSFO, as the nominal Fe valence scales linearly with $x$, assuming $\delta \approx 0$ in the oxygen stoichiometric films and that any cation-site deficiency is charge balanced by oxygen vacancies. The effective oxygen content in the oxygen deficient LSFO sample was estimated using this relationship between nominal Fe valence and absorption coefficient at $1.25 \mathrm{eV}$. Figure 3(b) shows the nominal Fe valence and oxygen deficiency as a function of heating time. Nominal Fe valence is estimated from the absorption coefficient in Figure $3(\mathrm{a})$, and $\delta$ is calculated using cation stoichiometry measured by RBS and the requirement for charge neutrality in the material. Based on this method, the most dramatic changes in oxygen content occur within the first $10 \mathrm{~min}$ of heating, when the nominal Fe valence shifts from $3 \cdot 76+$ to $3.21+$, corresponding to $\delta=0$ to $\delta=0.28$, respectively. We caution that this process of nominal Fe valence estimation contains inherent sources of uncertainty, both in the assumption of the linear relationship between absorption and nominal Fe valence and in error associated with the fit in
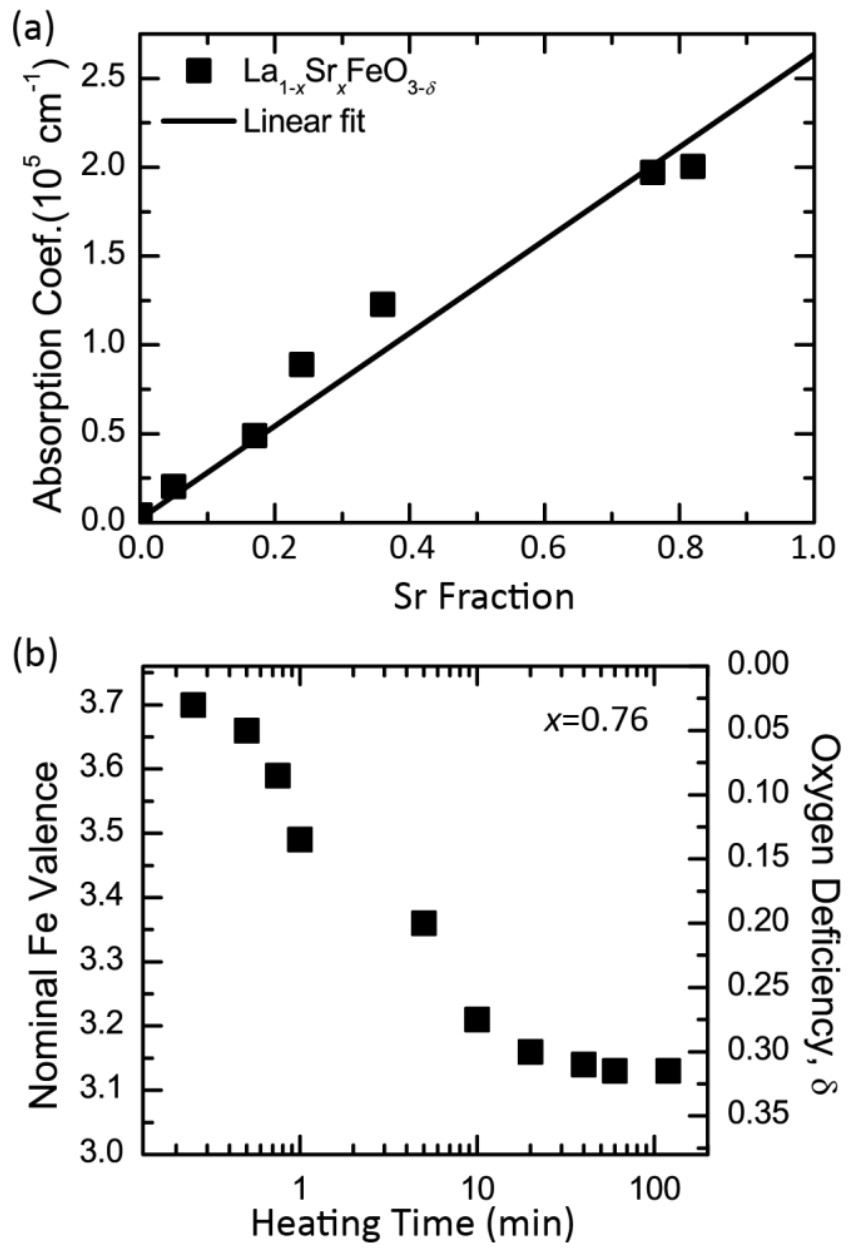

Figure 3. (a) Absorption coefficient versus $\mathrm{Sr}$ fraction in the oxygen stoichiometric $(\delta \approx 0)$ films. (b) Nominal Fe valence (left axis) and oxygen deficiency, $\delta$ (right axis) in the $x=0.76$ film as a function of heating time at $300^{\circ} \mathrm{C}$. In (a), nominal $\mathrm{Fe}$ valence is estimated as 3 plus the $\mathrm{Sr}$ fraction.

Figure 3(a). The statistical error, as determined from the fit in Figure $3(\mathrm{a})$, is $\sim 5.2 \%$; consequently, the value of $\delta$ carries an error of approximately \pm 0.08 . This error would uniformly shift the $\delta$ data in Figure $3(\mathrm{~b})$ to higher or lower $\delta$ values but the underlying trend of increasing $\delta$ with heating time would remain.

While the general trends in the absorption spectra shown in Figures 2(a) and 2(b) are similar, there are some differences in spectral features, particularly above $2.5 \mathrm{eV}$. These differences may arise from changes in Fe coordination number, which is reduced from 6 for $\delta=0$ to 4 or 5 for some Fe cations when $\delta \neq 0$. Additionally, even after two hours of heating, the nominal Fe valence in the $x=0.76$ film did not appear to reach a nominal Fe valence of $3^{+}$ but instead was closer to a nominal value of $3.1+$, based on the absorption coefficient at $1.25 \mathrm{eV}$. This is a likely explanation for why, in $x=0.76$, it was not possible to recover 
the two absorption features between 2.5 and $3.0 \mathrm{eV}$ found in pure LFO and why a low-energy absorption tail remains that is not present in pure LFO. We anticipate that films under a different strain state would exhibit different oxygen loss kinetics, as studies have shown a clear dependence between epitaxial strain and the formation of oxygen vacancies..$^{49-52}$ However, besides these differences, the absorption spectra achieved with either $A$-site substitution or oxygen vacancies are quite similar, despite differences in Fe coordination.

\subsection{Dynamic optoelectronic properties}

Changes with nominal Fe valence in UV-visible spectrum. Photoexcited electrons and holes produce characteristic modulations in the refractive index due to bandfilling. ${ }^{53}$ These changes are sensitive to electronic band structure and the energetic location of the carriers in the semiconductor. In ultrafast transient reflectance spectroscopy, such modulations manifest as reflectance changes which can be monitored with respect to both energy and pump-probe delay time to quantify the relaxation of photoexcited carriers.

UV-vis transient reflectance spectra are shown in Figure 4(a) for the oxygen stoichiometric LSFO films with different $\mathrm{Sr}$ fraction on LSAT. All the samples were pumped with an ultrafast, $4 \mathrm{eV}$ pulse and probed $5 \mathrm{ps}$ after photoexcitation. Several systematic trends in the transient reflectance spectra generally match observations from the VASE measurements. In undoped LFO, there are two negative reflectance transients at $\sim 2.4$ and $\sim 3.5 \mathrm{eV}$ that correspond to the onset of optical absorption. ${ }^{17}$ These negative differential reflectance features arise from transitions from the valence band to conduction band states dominated by the minority spin Fe $t_{2 g}$ and $e_{g}$ orbitals. As the $\mathrm{Sr}$ fraction in LSFO increases, these negative reflectance transients in the UV-vis range decrease in magnitude and broaden. This is consistent with the decrease of valence band majority spin $\mathrm{O} 2 p / \mathrm{Fe} e_{g}$ hybridized states as $\mathrm{Sr}$ fraction increases. In films with $x>0.5$, the negative reflectance transient disappears and becomes positive. Positive reflectance transients can arise from heating in semiconductors, ${ }^{54}$ perovskites, ${ }^{14}$ and metals,,${ }^{55}$ and may indicate the closing of the band gap in LSFO. Also, new absorption pathways within the conduction band can also give rise to increased differential reflectance.

Changes to the nominal Fe valence induced through the introduction of oxygen vacancies are also apparent in the transient reflectance spectra, as shown in Figure 4 (b). For the oxygen stoichiometric LSFO film, there is a characteristic broad positive reflectance transient, which is attributed to transitions that involve $\mathrm{Fe}^{4+}$ states (hybridized $\mathrm{O} 2 p$ /majority spin Fe $e_{g}$ states above the Fermi level). As the nominal Fe valence is reduced, this positive reflectance transient decreases in magnitude as the negative reflectance change originating from the transition between the valence band and the minority spin Fe states in the conduction band begins to dominate the observed spectral signal. As with the static absorption, films heated for the longest times approach, but do not quite reach, the spectral shape of pure $\mathrm{LaFeO}_{3}$.
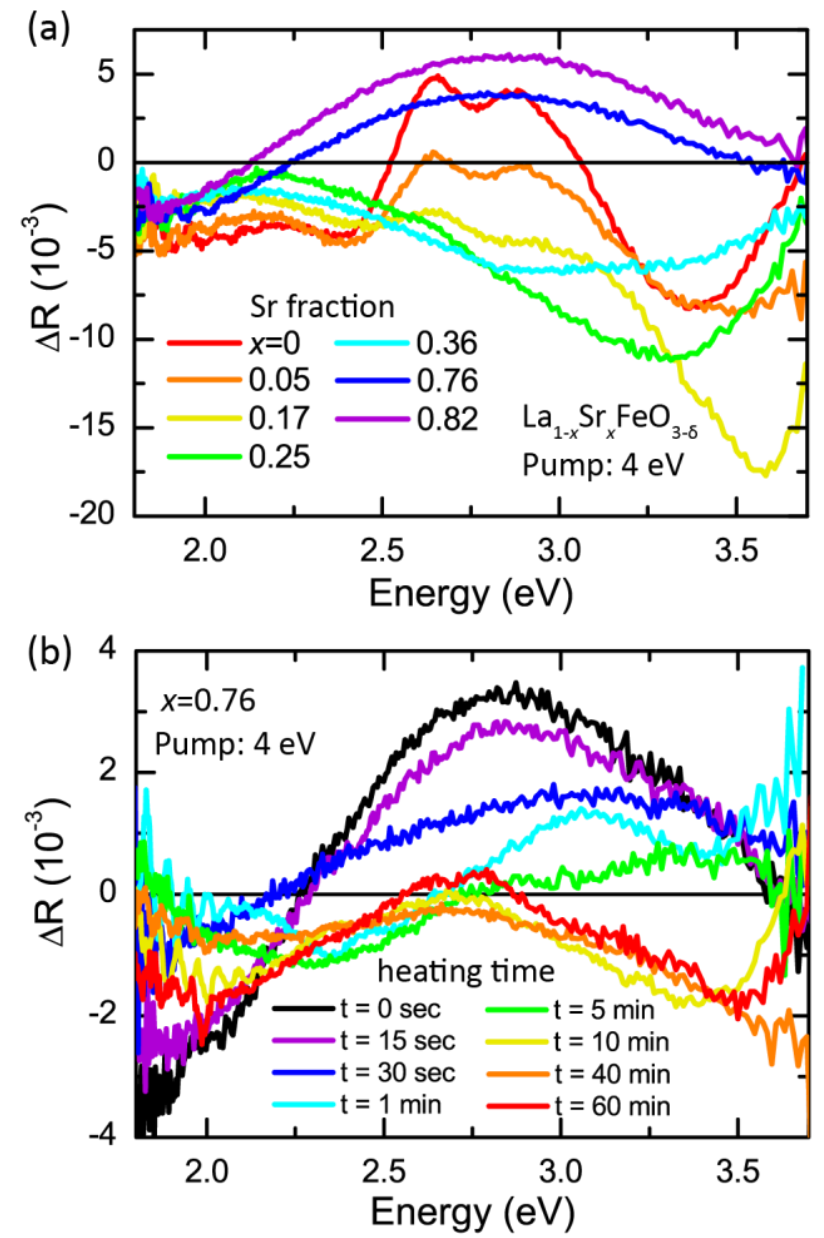

Figure 4. Transient reflectance spectra taken 5 ps following photoexcitation for LSFO films with (a) varying $\mathrm{Sr}$ fraction $(\delta \approx 0)$ and (b) oxygen vacancy concentration. Pump at 4.0 $\mathrm{eV}, 2.4 \mathrm{~mJ} / \mathrm{cm}^{2}$ (oxygen stoichiometric experiments) and $2.7 \mathrm{~mJ} / \mathrm{cm}^{2}$ (oxygen deficient experiments). In (a), nominal Fe valence is estimated as 3 plus the $\mathrm{Sr}$ fraction. The nominal Fe valence associated with the heating times in (b) can be seen in Figure 3 (b).

Near-infrared (NIR) probe of $\mathrm{Fe}^{4+}$ electronic configuration. The NIR region is sensitive to low-energy transitions, such as those that arise from the states related to $\mathrm{Fe}^{4+}$. Here we focus on NIR transient reflectance response of films whose nominal Fe valence was tuned with both $A$-site substitution and oxygen stoichiometry. Figure $5(\mathrm{a})$ and (b) show the differential reflectance in the NIR for the oxygen stoichiometric and oxygen deficient films, respectively, at a pump-probe delay time of $5 \mathrm{ps}$. It is important to note that in the oxygen deficient films, although the same heating procedure was used as with the visible ultrafast reflectance experiments, we do not claim that the oxygen content is the same as modeled in 

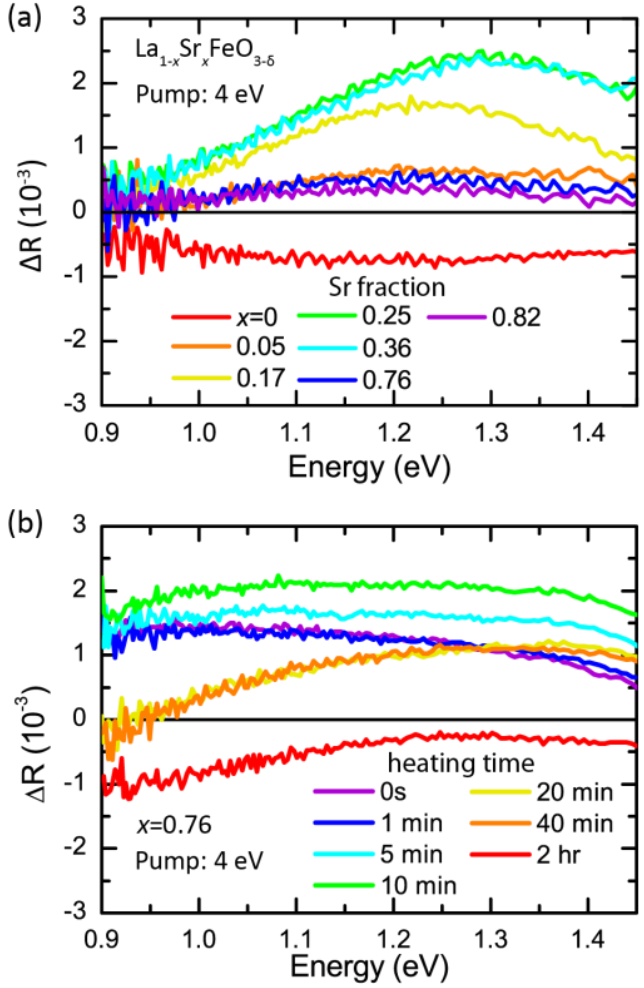
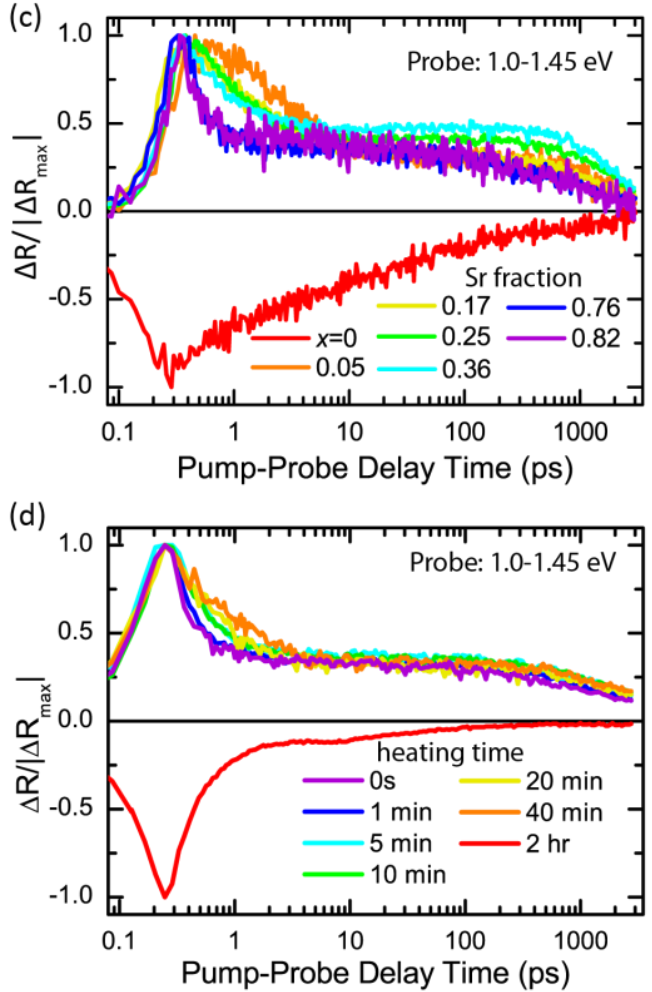

Figure 5. Near-infrared transient reflectance spectroscopy for LSFO films with (a,c) stoichiometric oxygen ( $\delta \approx 0)$ but varying Sr fraction, and (b,d) LSFO $x=0.76$ with varying oxygen deficiency. (a) and (b) show spectral data at 5 ps following photoexcitation. (c) and (d) show kinetics integrated over probed energies of 1.0-1.45 eV. The pump photon energy was $4.0 \mathrm{eV}$ and fluence was $0.12 \mathrm{~mJ} / \mathrm{cm}^{2}$. In $(\mathrm{a}, \mathrm{c})$, nominal Fe valence is estimated as 3 plus the $\mathrm{Sr}$ fraction.

Figure 3(b). Because we could not take corresponding ellipsometry data with the NIR experiments, we cannot estimate oxygen content, although with increasing heating time it is clear that oxygen vacancies are formed.

In both sets of samples, when films have a nominal $\mathrm{Fe}$ valence close to $3^{+}$, such as in LFO and very oxygen deficient LFSO $x=0.76$, there is a small negative change in reflectance. As the nominal Fe valence increases, it creates majority spin Fe $e_{g}$ conduction band states at the expense of analogous states in the valence band, giving rise to a positive reflectance change in the NIR region. The magnitude of the NIR reflectance transient depends on the joint density of conduction band and valence band states. When the density of majority spin Fe $e_{g} / \mathrm{O} 2 \mathrm{p}$ states in either the conduction band or valence band is low, the reflectance transient is small, as is the case for $x=0.05$ and 0.76 . The reflectance transient is maximized near $x=0.3$, indicating a maximum in the joint density of states at that composition. This conclusion is consistent with the work of Wadati et al., who found that the spectral weight of majority spin $\mathrm{Fe} e_{g} / \mathrm{O} 2 \mathrm{p}$ valence band states decreased superlinearly with increasing $\mathrm{Sr}$ fraction, while the conduction band states increased approximately linearly with $x$. This implies that the product of conduction and valence band states derived from majority spin $e_{g}$ states is maximized near $x=0.3-0.4$ in LSFO. ${ }^{36}$ Similar trends in the magnitude of the reflectance transient with increasing oxygen deficiency in the $x=0.76$ film are observed as with $\mathrm{Sr}$ alloying.

To understand the dynamic properties of the photoexcited carriers associated with $\mathrm{Fe}^{4+}$-induced transitions, the reflectance transients were monitored with time, as shown in Figure 5(b). The time delay following excitation varied between fs and a few ns, giving carrier dynamics on timescales relevant for recombination. The kinetics shown in Figure 5(b) were analyzed by integrating the spectral signal from 1.0-1.45 eV, thereby including the largest number of carriers contributing to the signal. The kinetics were normalized by the maximum differential reflectance to allow the temporal response between samples to be compared. The kinetics were fit with a biexponential decay model convoluted with a Gaussian instrument response:

$$
\frac{\Delta R}{\left|\Delta R_{\text {max }}\right|}=\left[A_{1} \exp \left(-\frac{t}{\tau_{1}}\right)+A_{2} \exp \left(-\frac{t}{\tau_{2}}\right)\right] \otimes G_{I R F}
$$

where the left-hand side is the normalized transient reflectance signal, $\boldsymbol{A}_{\mathbf{1}}$ and $\boldsymbol{A}_{\mathbf{2}}$ are the weights, and $\boldsymbol{\tau}_{\mathbf{1}}$ and $\boldsymbol{\tau}_{\mathbf{2}}$ are the fitted time constants, which are shown in Figure S2. Characteristic sample fits and residuals, are given in Figures $\mathrm{S}_{3}-\mathrm{S} 6$. 

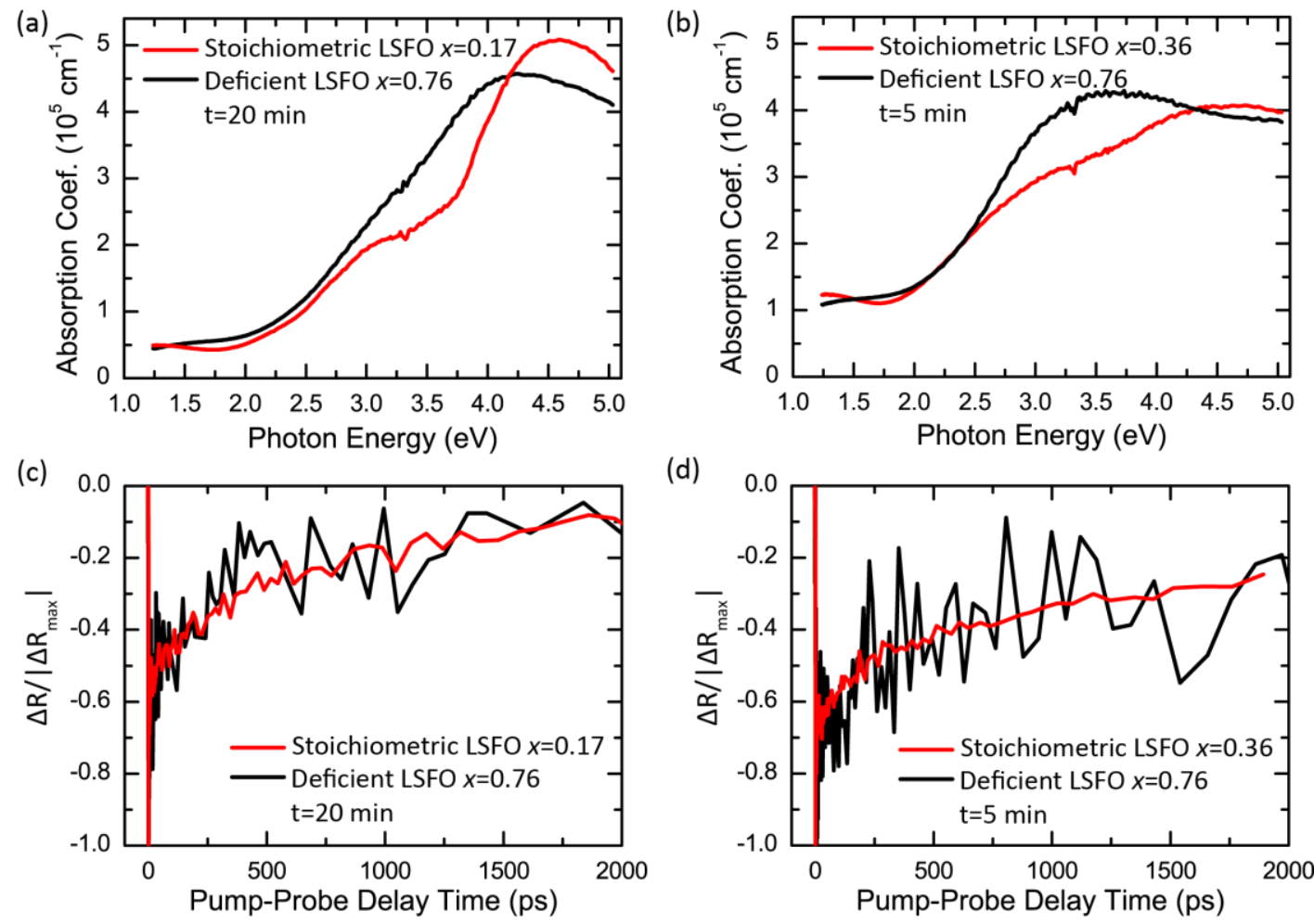

Figure 6. $(a, b)$ Comparison of optical absorption for LSFO films with same nominal Fe valence arrived through Sr fraction in oxygen-stoichiometric films and oxygen vacancies in LSFO $x=0.76$. (c,d) Corresponding comparison of carrier kinetics of LSFO. In oxygen stoichiometric and deficient samples, the main visible transient reflectance was integrated to arrive at the kinetics. The signal-to-noise in this sample was low due to the small magnitude of the reflectance change. Pump at $4.0 \mathrm{eV}, 2.4 \mathrm{~mJ} / \mathrm{cm}^{2}$ (oxygen stoichiometric experiments) and $2.7 \mathrm{~mJ} / \mathrm{cm}^{2}$ (oxygen deficient experiments).

When probing the NIR region in oxygen stoichiometric LSFO, decay on the order of a few picoseconds becomes faster with increasing $\mathrm{Sr}$ fraction, as shown in Figure 5(c) and Figure S2. Moreover, similar changes are observed with oxygen deficient LSFO $x=0.76$, as shown in Figure 5(d). In both cases, early time kinetics become faster as nominal Fe valence increases. Several physical phenomena could give rise to dynamics on this time scale $\left(\tau_{1}\right)$, including Auger recombination, carrier thermalization, and trapping. All of these phenomena might be expected to depend on the density of $\mathrm{Fe}^{4^{+}}$-induced states above the Fermi level. A similar effect has been observed in $\mathrm{SrTiO}_{3}$ doped with $\mathrm{La}$ and $\mathrm{Cr}^{23}$

The kinetics of the nanosecond-scale decay did not vary dramatically as a function of $A$-site composition for $x<0.5$, while the compositions with $x>0.5$ exhibited slightly faster kinetics, as shown by the fitted $\tau_{2}$ values in Figure $\mathrm{S}_{2}$ and raw kinetics in Figure 5(c). In oxygen-deficient films, The long time constant was $\sim 2$ ns and was relatively independent of oxygen content before increasing to $3 \mathrm{~ns}$ at longer heating times. Based on our previous work on $\mathrm{LaFeO}_{3}{ }^{17}$ we suspect that this longer recombination lifetime can be associated with a recombination mechanism mediated by trap states, such as Shockley-Read-Hall or surface recombination. Other ultrafast experiments on perovskite oxides have attributed nanosecond scale time constants to trapping into localized states, ${ }^{16}$ and radiative recombination. ${ }^{15}$

Similar lifetimes achieved by manipulating $\boldsymbol{A}$-site and oxygen composition. The ability to alter nominal Fe valence through oxygen vacancies allows us to probe the effects of anion point defects on recombination lifetimes by comparing to oxygen stoichiometric films with the same nominal Fe valence. Figure 6 shows a direct comparison of static and dynamic optical properties for samples of similar nominal Fe valence, $\sim 3.2+$ in $(\mathrm{a}, \mathrm{c})$ and $\sim 3.4+$ in (b,d), but achieved through $A$-site substitution or oxygen composition. The similarity between absorption coefficients and onsets at low energies observed with increasing $\delta$ in the $x=0.76$ sample and that obtained from the films with decreasing $x$ is apparent. The kinetics of the $x=0.17 \quad(\delta \approx 0)$ and the $x=0.76 \quad(\delta \approx 0.3)$ samples, each with nominal Fe valence of $\sim \mathbf{3 . 2 +}$, are compared in Figure 6(c). The similarity of the kinetic traces is also evident. The kinetics of each film can be fit with a biexponential decay function with a long time constant $\left(\tau_{2}\right)$ of $870 \pm 40 \mathrm{ps}$ and $930 \pm 170$ ps for the stoichiometric and deficient films, respectively. Similar agreement is found with the $x=0.36$ $(\delta \approx 0)$ and the $x=0.76(\delta \approx 0.2)$ films, Figure $6(d)$, which have $\tau_{2}$ of $1710 \pm 120$ ps and $1790 \pm 260$ ps, respectively. 
Physically, this result indicates that the nominal Fe valence, achieved through either cation composition or anion vacancies, is a critical factor for photoexcited carrier lifetimes in ferrite perovskites. The increased concentration of anion vacancies in the oxygen deficient film does not significantly enhance or reduce carrier lifetimes, a surprising result given the role that defects commonly play in altering recombination kinetics. ${ }^{56}$ Our findings point to the need for a deeper understanding of the fundamental mechanisms that limit recombination times in semiconducting perovskite oxides.

\section{CONCLUSIONS}

We have demonstrated how both heterovalent $A$-site substitution and oxygen vacancies can be used to control optical absorption in $\mathrm{La}_{1-x} \mathrm{Sr}_{x} \mathrm{FeO}_{3-\delta}$ perovskite films. Both routes of changing the nominal Fe valence produced similar optical absorption spectra. We find that the nominal Fe valence is proportional to the absorption coefficient near $1.25 \mathrm{eV}$ providing a means to estimate the oxygen deficiency of oxygen deficient LSFO. Ultrafast transient reflectance spectroscopy corroborated the optical absorption data, showing the systematic creation and population of excited states that depend on the nominal Fe valence state. The effect of oxygen vacancies on carrier kinetics was investigated by varying the oxygen content in a single $\mathrm{La}_{0.24} \mathrm{Sr}_{0.76} \mathrm{FeO}_{3-\delta}$ film. Ultrafast spectroscopy in the NIR on oxygen deficient films revealed analogous trends as the oxygen stoichiometric films with varying $\mathrm{Sr}$ fraction. Surprisingly, carrier dynamics probed in the visible range of the oxygen deficient film were quite similar to oxygen stoichiometric films with similar nominal Fe valence. These results indicate that cation and anion stoichiometry can be used to alter optical absorption in ferrites, with both approaches leading to similar photoexcited carrier dynamics.

\section{ASSOCIATED CONTENT}

Supporting Information Available: Additional data of optical absorption and transient reflectance time constants and fits. This material is available free of charge via the Internet at http://pubs.acs.org.

\section{AUTHOR INFORMATION}

\section{Corresponding Author}

* (S.J.M.). E-mail: smay@coe.drexel.edu

\section{Author Contributions}

All authors have given approval to the final version of the manuscript. ¥These authors (S.Y.S and M.D.S) contributed equally.

\section{Notes}

The authors declare no competing financial interest.

\section{ACKNOWLEDGMENT}

The authors acknowledge support from the National Science Foundation through grant ECCS-1201957. The ultra- fast spectrometer with UV-vis probe at Drexel University was acquired with funds from the NSF MRI award DMR0922929. The x-ray diffractometer was acquired with funds from NSF MRI award DMR-1040166. We thank Boris Yakshinskiy for RBS measurements at the Rutgers University Laboratory for Surface Modification. This research used resources of the Center for Functional Nanomaterials, which is a U.S. DOE Office of Science Facility, at Brookhaven National Laboratory under Contract No. DESCoo12704.

\section{REFERENCES}

(1) Peña, M. A.; Fierro, J. L. G. Chemical Structure and Performance of Perovskite Oxides. Chem. Rev. 2001, 101, 1981-2017.

(2) Goodenough, J. B. Electronic and Ionic Transport Properties and Other Aspects of Perovskites. Rep. Prog. Phys. 2004, 67, 1915-1993.

(3) Dagotto, E. Complexity in Strongly Correlated Electron Systems. Science 2005, 309, 257-262.

(4) Apgar, B. A.; Lee, S. K.; Schroeder, L. E.; Martin, L. W. Enhanced Photoelectrochemical Activity in All-Oxide Heterojunction Devices Based on Correlated "Metallic" Oxides. Adv. Mater. 2013, 25, 6201-6206.

(5) Liu, J. W.; Chen, G.; Li, Z. H.; Zhang, Z. G. Electronic Structure and Visible Light Photocatalysis Water Splitting Property of Chromium-Doped $\mathrm{SrTiO}_{3}$. J. Solid State Chem. 2006, 179, 3704-3708.

(6) Castelli, I. E.; Olsen, T.; Datta, S.; Landis, D. D.; Dahl, S.; Thygesen, K. S.; Jacobsen, K. W. Computational Screening of Perovskite Metal Oxides for Optimal Solar Light Capture. Energy Environ. Sci. 2012, 5, 5814-5819.

(7) Yang, S. Y.; Seidel, J.; Byrnes, S. J.; Shafer, P.; Yang, C. H.; Rossell, M. D.; Yu, P.; Chu, Y. H.; Scott, J. F.; Ager, J. W.; Martin, L. W.; Ramesh, R. Above-Bandgap Voltages from Ferroelectric Photovoltaic Devices. Nat. Nanotechnol. 2010, 5, 143-148.

(8) Grinberg, I.; West, D. V.; Torres, M.; Gou, G. Y.; Stein, D. M.; Wu, L. Y.; Chen, G. N.; Gallo, E. M.; Akbashev, A. R.; Davies, P. K.; Spanier, J. E.; Rappe, A. M. Perovskite Oxides for Visible-Light-Absorbing Ferroelectric and Photovoltaic Materials. Nature 2013, 503, 509-512.

(9) Assmann, E.; Blaha, P.; Laskowski, R.; Held, K.; Okamoto, S.; Sangiovanni, G. Oxide Heterostructures for Efficient Solar Cells Phys. Rev. Lett. 2013, 110, 078701.

(10) Wang, L.; Li, Y.; Bera, A.; Ma, C.; Jin, F.; Yuan, K.; Yin, W.; David, A.; Chen, W.; Wu, W.; Prellier, W.; Wei, S.; $\mathrm{Wu}, \mathrm{T}$. Device Performance of the Mott Insulator $\mathrm{LaVO}_{3}$ as a Photovoltaic Material. Phys. Rev. Appl. 2015, 3, 064015.

(11) Zhang, G.; Wu, H.; Li, G.; Huang, Q.; Yang, C.; Huang, F.; Liao, F.; Lin, J. New High $T_{C}$ Multiferroics $\mathrm{KBiFe}_{2} \mathrm{O}_{5}$ with Narrow Band Gap and Promising Photovoltaic Effect. Sci. Rep. 2013, 3, 1265.

(12) Porter, S. H.; Huang, Z.; Doe, S.; Brown-Xu, S.; Golam Sarwar, A. T. M.; Myers, R. C.; Woodward, P. M. Electronic Structure and Photocatalytic Water Oxidation Activity of $R \mathrm{TiNO}_{2}(R=\mathrm{Ce}, \mathrm{Pr}$, and $\mathrm{Nd})$ Perovskite Nitride Oxides. Chem. Mater. 2015, 27, 2414-2420. 
(13) Arima, T.; Tokura, Y.; Torrance, J. B. Variation of Optical Gaps in Perovskite-Type $3 d$ Transition-Metal Oxides. Phys. Rev. B 1993, 48, 17006-17010.

(14) Eng, H. W.; Barnes, P. W.; Auer, B. M.; Woodward, P. M. Investigations of the Electronic Structure of $d^{\circ}$ Transition Metal Oxides Belonging to the Perovskite Family. J. Solid State Chem. 2003, 175, 94-109.

(15) Sheu, Y. M.; Trugman, S. A.; Park, Y. S.; Lee, S.; Yi, H. T.; Cheong, S. W.; Jia, Q. X.; Taylor, A. J.; Prasankumar, R. P. Ultrafast Carrier Dynamics and Radiative Recombination in Multiferroic $\mathrm{BiFeO}_{3}$. Appl. Phys. Lett. 2012, 100, 242904.

(16) Yamada, Y.; Nakamura, T.; Yasui, S.; Funakubo, H.; Kanemitsu, Y. Meauurement of Transient Photoabsorption and Photocurrent of $\mathrm{BiFeO}_{3}$ Thin Films: Evidence for Long-Lived Trapped Photocarriers. Phys. Rev. B 2014, 89, 035133.

(17) Smolin, S. Y.; Scafetta, M. D.; Guglietta, G. W.; Baxter, J. B.; May, S. J. Ultrafast Transient Reflectance of Epitaxial Semiconducting Perovskite Thin Films. Appl. Phys. Lett. 2014, 105, 022103.

(18) Hwang, H. Y.; Iwasa, Y.; Kawasaki, M.; Keimer, B.; Nagaosa, N.; Tokura, Y. Emergent Phenomena at Oxide Interfaces. Nature Mater. 2012, 11, 103-113.

(19) Rondinelli, J. M.; May, S. J.; Freeland, J. W. Control of Octahedral Connectivity in Perovskite Oxide Heterostructures: An Emerging Route to Multifunctional Materials Discovery. MRS Bull. 2012, 37, 261-270.

(20) Baxter, J. B.; Richter, C.; Schmuttenmaer, C. A. Ultrafast Carrier Dynamics in Nanostructures for Solar Fuels. Annu. Rev. Phys. Chem. 2014, 65, 423-447.

(21) Jin, Z.; Xu, Y.; Zhang, Z.; Li, G.; Lin, X.; Ma, G.; Cheng, Z.; Wang, X. Structural Dependent Ultrafast Elec-

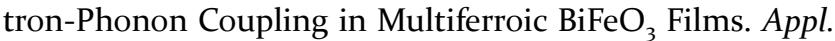
Phys. Lett. 2012, 100, 071105.

(22) Li, Z.-G.; Zhao, R.; Li, W.; Wang, H.; Yang, H.; Song, Y.-L. Strain Dependent Ultrafast Carrier Dynamics in Eu$\mathrm{TiO}_{3}$ Films. Appl. Phys. Lett. 2014, 105, 162904.

(23) Comes, R. B.; Smolin, S. Y.; Kaspar, T. C.; Gao, R.; Apgar, B. A.; Martin, L. W.; Bowden, M. E.; Baxter, J. B.; Chamber, S. A. Visible Light Carrier Generation in CoDoped Epitaxial Titanate Films. Appl. Phys. Lett. 2015, 106, 092901.

(24) Scafetta, M. D.; Cordi, A. M; Rondinelli, J. M.; May, S. J. Band Structure and Optical Transitions in $\mathrm{LaFeO}_{3}$ : Theory and Experiment. J. Phys.: Condens. Matter 2014, 26, 505502 .

(25) Fossdal, A.; Menon, M.; Wærnhus, I.; Wiik, K.; Einarsrud, M.-A.; Grande, T. Crystal Structure and Thermal Expansion of $\mathrm{La}_{1-x} \mathrm{Sr}_{x} \mathrm{FeO}_{3-\delta}$ Materials. J. Am. Ceram. Soc. 2004, 87, 1952-1958.

(26) Clemens, O.; Kuhn, M.; Haberkorn, R. Synthesis and Characterization of the $\mathrm{La}_{1-x} \mathrm{Sr}_{x} \mathrm{FeO}_{3-\delta}$ System and the Fluorinated Phases of $\mathrm{La}_{1-x} \mathrm{Sr}_{x} \mathrm{FeO}_{3-x} \mathrm{~F}_{x}$. J. Solid State Chem. 2011, 184, 2870-2876.

(27) Abbate, M.; de Groot, F. M. F.; Fuggle, J. C.; Fujimori, A.; Strebel, O.; Lopez, F.; Domke, M.; Kaindl, G.;
Sawatzky, G. A.; Takano, M.; Takeda, Y.; Eisaki, H.; Uchida, S. Controlled-Valence Properties of $\mathrm{La}_{1-x} \mathrm{Sr}_{x} \mathrm{FeO}_{3}$ and $\mathrm{La}_{1-}$ ${ }_{x} \mathrm{Sr}_{x} \mathrm{MnO}_{3}$ Studied by Soft X-ray Absorption Spectroscopy. Phys. Rev. B 1992, 46, 4511-4519.

(28) Wadati, H.; Chikamatsu, A.; Takizawa, M.; Hashimoto, R.; Kumigashira, H.; Yoshida, T.; Mizokawa, T.; Fujimori, A.; Oshima, M.; Lippmaa, M.; Kawasaki, M.; Koinuma, H. Strong Localization of Doped Holes in $\mathrm{La}_{1-}$ ${ }_{x} \mathrm{Sr}_{x} \mathrm{FeO}_{3}$ from Angle-Resolved Photoemission Spectra. Phys. Rev. B 2006, 74, 115114.

(29) Maeder, T.; Bednorz, J. G. Influence of Oxygen Stoichiometry on Electrical Transport and Magnetic Properties of Doped Perovskite-type Ferrate and Manganate Single Crystals. J. Euro. Ceram. Soc. 1999, 19, 1507-1510.

(30) Xie, Y. J.; Scafetta, M. D.; Moon, E. J.; Krick, A. L.; Sichel-Tissot, R. J.; May, S. J. Electronic Phase Diagram of Epitaxial $\mathrm{La}_{1-x} \mathrm{Sr}_{x} \mathrm{FeO}_{3}$ Films. Appl. Phys. Lett. 2014, 105 o62110.

(31) Battle, P. D.; Gibb, T. C.; Lightfoot, P. The Structural Consequences of Charge Disproprotionation in MixedValence Iron Oxides. I. The Crystal Structure of $\mathrm{Sr}_{2} \mathrm{LaFe}_{3} \mathrm{O}_{8.94}$ at Room Temperature and 50 K. J. Solid State Chem. 1990, 84, 271-279.

(32) Park, S. K.; Ishikawa, T.; Tokura, Y.; Li, J. Q.; Matsui, $\mathrm{Y}$. Variation of Charge-Ordering Transitions in $R_{1 / 3} \mathrm{Sr}_{2 / 3} \mathrm{FeO}_{3}(R=\mathrm{La}, \mathrm{Pr}, \mathrm{Nd}, \mathrm{Sm}$, and Gd). Phys. Rev. $B$ 1999, 6o, 10788-10795.

(33) Okamoto, J.; Huang, D. J.; Chao, K. S.; Huang, S. W.; Hsu, C.-H.; Fujimori, A.; Masuno, A.; Terashima, T.; Takano, M.; Chen, C. T. Quasi-Two-Dimensional $d$-Spin and $p$ Hole Ordering in the Three-Dimensional Perovskite $\mathrm{La}_{1 / 3} \mathrm{Sr}_{2 / 3} \mathrm{FeO}_{3}$. Phys. Rev. B 2010, 82, 132402.

(34) Devlin, R. C.; Krick, A. L.; Sichel-Tissot, R. J.; Xie, Y. J.; May, S. J. Electronic Transport and Conduction Mechanism Transition in $\mathrm{La}_{1 / 3} \mathrm{Sr}_{2 / 3} \mathrm{FeO}_{3}$ Thin Films. J. Appl. Phys. 2014, 115233704.

(35) MacChesney, J. B.; Sherwood, R. C.; Potter, J. F. Electric and Magnetic Properties of the Strontium Ferrates. J. Chem. Phys. 1965, 43, 1907-1913.

(36) Wadati, H.; Kobayashi, D.; Kumigashira, H.; Okazaki, K.; Mizokawa, T.; Fujimori, A.; Horiba, K.; Oshima, M.; Hamada, N.; Lippmaa, M.; Kawasaki, M.; Koinuma, H. Hole-Doping-Induced Changes in the Electronic Structure of $\mathrm{La}_{1-x} \mathrm{Sr}_{x} \mathrm{FeO}_{3}$ : Soft X-ray Photoemission and Absorption Study of Epitaxial Thin Films. Phys. Rev. B 2005, 71, 035108.

(37) Scafetta, M. D.; Xie, Y. J.; Torres, M.; Spanier, J. E.; May, S. J. Optical Absorption in Epitaxial $\mathrm{La}_{1-x} \mathrm{Sr}_{x} \mathrm{FeO}_{3}$ Thin Films. Appl. Phys. Lett. 2013, 102, 081904.

(38) Ritzmann A. M.; Muñoz-Garcia, A. B.; Pavone, M.; Keith, J. A.; Carter, E. A. Ab Initio DFT+U Analysis of Oxygen Vacancy Formation and Migration in $\mathrm{La}_{1-x} \mathrm{Sr}_{x} \mathrm{FeO}_{3-\delta}$ ( $x=0,0.25,0.50)$. Chem. Mater. 2013, 25, 3011-3019.

(39) Hodges, J. P.; Short, S.; Jorgensen, J. D.; Xiong, X.; Dabrowski, B.; Mini, S. M.; Kimball, C. W. Evolution of Oxygen-Vacancy Ordered Crystal Structures in the Perovskite Series $\mathrm{Sr}_{n} \mathrm{Fe}_{n} \mathrm{O}_{3 n-1}(n=2,4,8$, and $\infty)$, and the Rela- 
tionship to Electronic and Magnetic Properties. J. Solid State Chem. 2000, 151, 190-209.

(40) Søgaard, M.; Vang Hendriksen, P.; Mogensen, M. Oxygen Nonstoichiometry and Transport Properties of Strontium Substituted Lanthanum Ferrite. J. Solid State Chem. 2007, 180, 1489-1503.

(41) Chen, X.; Grande, T. Anisotropic and Nonlinear Thermal and Chemical Expansion of $\mathrm{La}_{1-x} \mathrm{Sr}_{x} \mathrm{FeO}_{3-\delta}(x=0.3$, 0.4, 0.5). Chem. Mater. 2013, 25, 3296-3306.

(42) Adler, P.; Lebon, A.; Damljanović, V.; Ulrich, C.; Bernhard, C.; Boris, A. V.; Maljuk, A.; Lin, C. T.; Keimer, B. Magnetoresistance Effects in $\mathrm{SrFeO}_{3-\delta}$ : Dependence on Phase Composition and Relation to Magnetic and Charge Order. Phys. Rev. B 20o6, 73, 094451.

(43) Xie, Y.; Scafetta, M. D.; Sichel-Tissot, R. J.; Moon, E. J.; Devlin, R. C.; Wu, H.; Krick, A. L.; May, S. J. Control of Functional Responses Via Reversible Oxygen Loss in $\mathrm{La}_{1-}$ ${ }_{x} \mathrm{Sr}_{x} \mathrm{FeO}_{3-\delta}$ Films. Adv. Mater. 2014, 26, 1434-1438

(44) Yamada, H.; Kawasaki, M.; Tokura, Y. Epitaxial Growth and Valence Control of Strained Perovskite $\mathrm{SrFeO}_{3}$ Films. Appl. Phys. Lett. 2002, 8o, 622-624.

(45) Björck, M.; Andersson, G. GenX: An Extensible Xray Reflectivity Refinement Program Utilizing Differential Evolution. J. Appl. Crystallogr. 2007, 40, 1174-1178.

(46) Mizusaki, J.; Yoshihiro, M.; Yamauchi, S.; Fueki, K. Nonstoichiometry and Defect Structure of the PerovskiteType Oxides $\mathrm{La}_{1-x} \mathrm{Sr}_{x} \mathrm{FeO}_{3-\delta}$. J. Solid State Chem. 1985, 58, 257-266.

(47) Cheng, J.; Navrotsky, A.; Zhou, X.-D.; Anderson, H. U. Thermochemistry of $\mathrm{La}_{1-x} \mathrm{Sr}_{x} \mathrm{FeO}_{3-\delta}$ Solid Solutions (o.o $\leq x \leq 1.0$, o.o $\leq \delta \leq 0.5$ ).Chem. Mater. 2005, 17, 2197-2207.

(48) Ishikawa, T.; Park, S. K.; Katsufuji, T.; Arima, T.; Tokura, Y. Optical Spectroscopy of Charge-Ordering Transition in $\mathrm{La}_{1 / 3} \mathrm{Sr}_{2 / 3} \mathrm{FeO}_{3}$. Phys. Rev. B 1998, 58, R13326Ri3329.

(49) Klenov, D. O.; Donner, W.; Foran, B.; Stemmer, S. Impact of stress on oxygen vacancy ordering in epitaxial $\left(\mathrm{La}_{0.5} \mathrm{Sr}_{0.5}\right) \mathrm{CoO}_{3-\mathrm{d}}$ thin films. Appl. Phys. Lett. 2003, 82, 3427-3429.

(50) Donner, W.; Chen, C. L.; Liu, M.; Jacobson, A. J.; Lee, Y.-L.; Gadre, M.; Morgan, D. Epitaxial Strain-Induced Chemical Ordering in $\mathrm{La}_{0.5} \mathrm{Sr}_{0.5} \mathrm{CoO}_{3-\mathrm{d}}$ Films on $\mathrm{SrTiO}_{3}$. Chem. Mater. 2011, 23, 984-988.

(51) Gazquez, J.; Bose, S.; Sharma, M.; Torija, M. A.; Pennycook, S. J.; Leighton, C.; Valera, M. Lattice mismatch accommodation via oxygen vacancy ordering in epitaxial $\mathrm{La}_{0.5} \mathrm{Sr}_{0.5} \mathrm{CoO}_{3-\mathrm{d}}$ thin films. APL Mater. 2013, 1, 012105.

(52) Aschauer, U.; Pfennginer, R.; Selbach, S. M.; Grande, T.; Spaldin, N. A.; Strained-controlled oxygen vacancy formation and ordering in $\mathrm{CaMnO}_{3}$. Phys. Rev. B 2013, 88, 054111.

(53) Bennett, B. R.; Soref, R. A.; Del Alamo, J. A. CarrierInduced Change in Refractive Index of InP, GaAs, and InGaAsP. IEEE J. Quantum Electron. 1990, 26, 113-122.

(54) Othonos, A.; van Driel, H. M.; Young, J. F.; Baribeau, J.-M. Free Carrier and Lattice-Heating-Induced
Changes to the Reflectivity of Epitaxial GeSi Alloys Following Picosecond Pulse Excitation. Solid State Commun. 1992, 82, 325-328.

(55) Miklós, A.; Bozóki, Z.; Lörincz, A. Picosecond Transient Reflectance of Thin Metal Films. J. Appl. Phys. 1989, 66, 2968-2972.

(56) Nelson, J. The Physics of Solar Cells; Imperial College Press: London, 2003. 


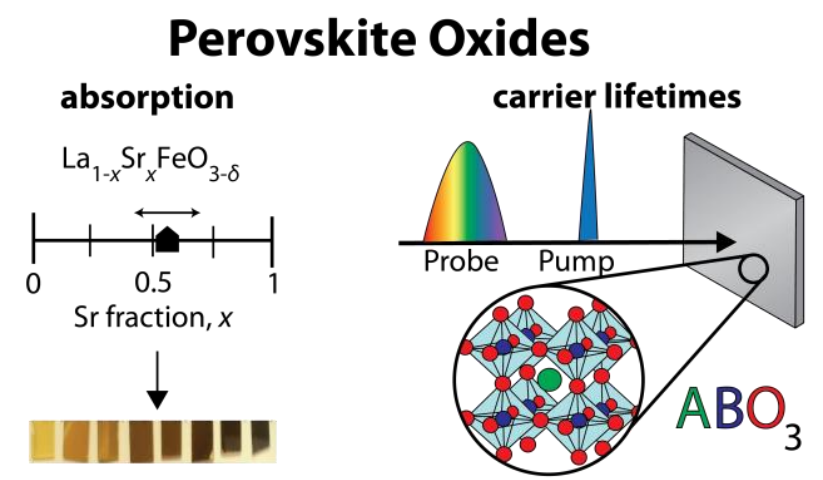

